\title{
Problems and Solutions of Velocity Modeling in Natural Gas Exploration
}

\author{
Aiqun Liu*, Caiwei Fan, Yong Deng, Peiyuan Zhu, Qianwei Hu, Peng Song \\ Zhanjiang Branch of China National Offshore Oil Corporation Ltd., Zhanjiang, China \\ Email: liuaq@cnooc.com.cn
}

How to cite this paper: Liu, A.Q., Fan, C.W., Deng, Y., Zhu, P.Y., Hu, Q.W. and Song, P. (2017) Problems and Solutions of Velocity Modeling in Natural Gas Exploration. Open Journal of Marine Science, 7, 51-61.

http://dx.doi.org/10.4236/ojms.2017.71005

Received: July 20, 2016

Accepted: December 3, 2016

Published: December 6, 2016

Copyright $\odot 2017$ by authors and Scientific Research Publishing Inc. This work is licensed under the Creative Commons Attribution International License (CC BY 4.0).

http://creativecommons.org/licenses/by/4.0/

\begin{abstract}
Recently, velocity prediction and modeling have been focus of the geophysical exploration in the high temperature and high pressure of the south China sea area, especially for new offshore exploratory areas. The error is large with great difficulty owing to fewer exploratory wells and misunderstanding. Firstly, on the basis of three typical velocity prediction and modeling examples in Ying-Qiong basin, it's easy to put forward the corresponding not common but urgent problem in each instance, then combined with the velocity problem and misunderstanding to expand method discussion and solution, which include geological model to guide the velocity interpretation and analysis, the establishment of forward velocity of the auxiliary model explaining and constructing high precision velocity model. This research basically solves existing velocity problems in gas exploration of south China sea in recent years, and proposes corresponding solution and application, which is of great significance to the further exploration and productive practice.
\end{abstract}

\section{Keywords}

Ying-Qiong Basin, Natural Gas Exploration, Velocity Modeling, Velocity Prediction, Velocity Analysis, Velocity Volume, The Geological Model, Inclination Correction, High Precision Velocity Model, Forward Modeling

\section{Introduction}

The west coast of South China Sea is rich in natural gas resource and has been an important natural gas exploration area for CNOOC. The Dong Fang gas field in Ying Gehai basin and the Ya Cheng gas field in Qiong Dong Nan basin are both large offshore natural gas field of China. However, in addition to the existing large gas fields put into operation, the overall prospecting degree of Ying-Qiong basin is low [1] [2] [3] [4]. It's very difficult to predict the velocity of favorable targets in a basin with low overall prospecting degree like Ying-Qiong basin. The reason is various. One reason is due to the low degree of prospecting, there are less exploratory wells and corresponding VSP 
velocity data, so it is difficult to understand the change trend of horizontal and vertical velocity. The other reason is the misunderstanding existed in velocity prediction process. And a third reason is because of the common problems encountered in the actual work.

In this paper, three typical cases of velocity forecast and model building in YingQiong basin are analyzed. And every case raises an unusual but pressing problem. There are three main problems: how to do velocity reprocessing and determine its accuracy, the system error existing between logging VSP velocity and velocity spectrum interpretation, and larger error occurs when predicting depth of new drilling well with the time-distance relationship of drilled wells nearby. Combined with problems above and the misunderstanding, method research is carried out and corresponding solution is put forward for every problem, which lays a solid foundation for accurate velocity prediction before drilling.

\section{Misunderstanding in the Velocity Prediction Process of Conventional Natural Gas Exploration}

As is known to all, velocity prediction is key and difficulty for the geophysical work. Conventionally, stacking velocity is obtained by velocity spectrum interpretation, and the accuracy of interpretation is judged by flattening effect of phase axis. Under the premise of formation with no large dip angle, the stacking velocity is equivalent to root mean square velocity. Meanwhile, when the seismic data is in good quality, velocity conversion formula (e.g. the DIX formula) can be used to convert root mean square velocity into interval velocity or the average velocity which can be used in pressure prediction and time-depth conversion [5] [6] [7]. But in practice, the assumptions that formation has no large dip angle and seismic data is in good quality are difficult to meet, and there also exists misunderstanding of velocity prediction [8]. So velocity prediction tends to have larger error.

1) Direct application of imaging velocity in time-depth conversion

This is the first misunderstanding of velocity prediction. Gathers data of the same velocity spectrum point are chosen from the deepwater area in the Qiong Dong Nan basin. Three different interpretations are made, including lesser velocity, medium velocity, and biggish velocity (Figure 1). Stacking velocity is obtained by velocity spectrum interpretation, and the accuracy of interpretation is judged by flattening effect of phase axis. We can see that the flattening effect of phase axes in three different solutions is basically consistent, but large errors (about $10 \%$ between low/high velocity and medium velocity) occur. So it is problematic to determine accuracy of velocity interpretation only using flattening effect of gathers data or imaging quality. Hereby, we come to the conclusion that the imaging velocity obtained through seismic processing usually cannot be directly used to make pressure prediction and time-depth conversion [9].

2) The establishment of velocity volume can improve time-depth conversion accuracy

Many people believe that establishing velocity volume can improve the accuracy of the time-depth conversion. The idea is very wrong. The velocity volume we get through 

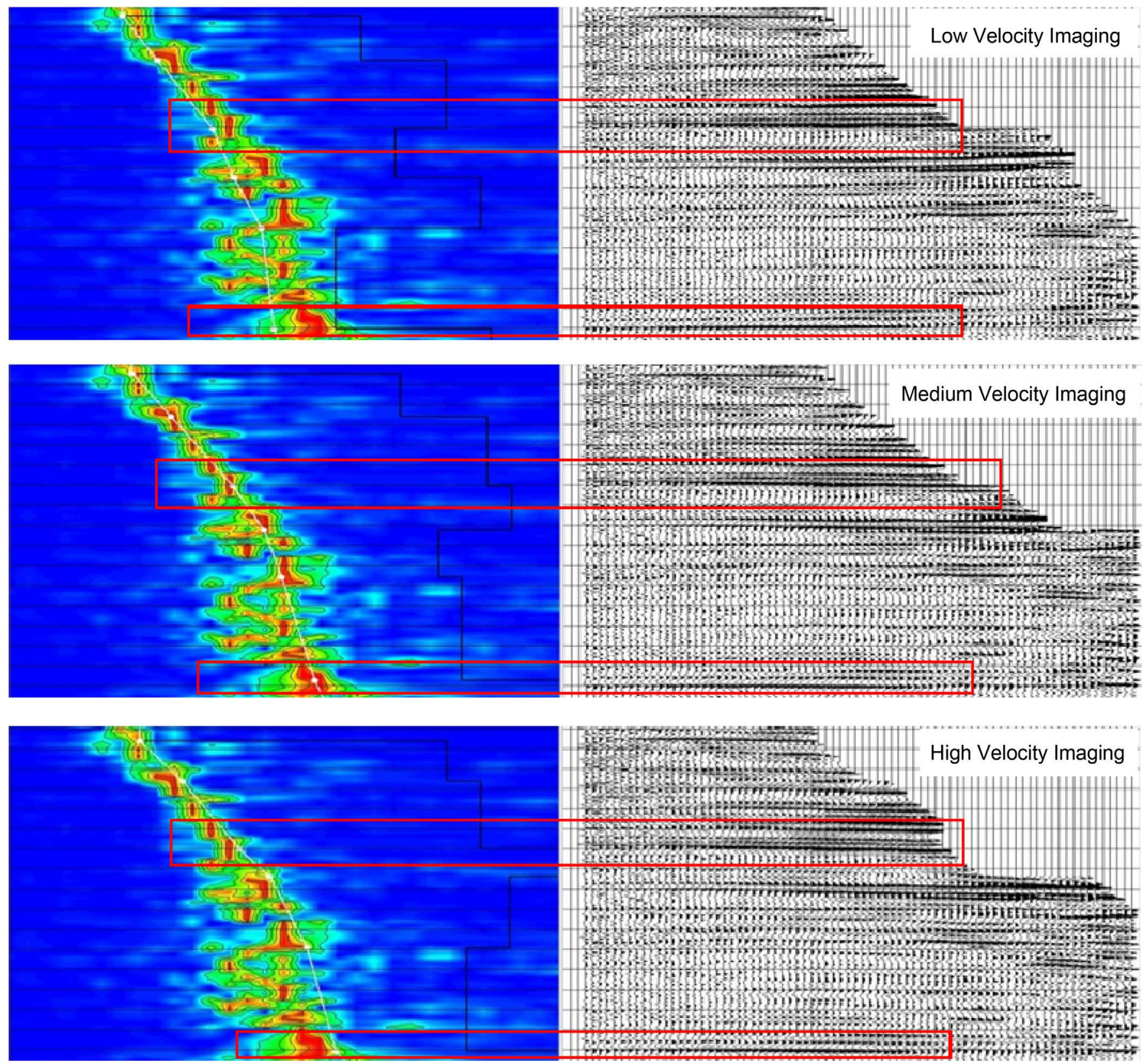

Figure 1. Three different interpretation schemes for the same velocity spectrum point.

whatever methods and software is based on single point gathers and velocity interpretation, and takes reference of the lateral variation trends of layers and longitudinal change trends of VSP data on the well site, and is obtained by the method of interpolation extrapolation. In this way, velocity accuracy of single-point velocity gathers, and velocity volume accuracy still depends upon single point velocity interpretation precision. Only on the premise of higher single point velocity interpretation precision could the data be used to build velocity volume and extract information of single point, layer and volume [10] [11] [12] (Figure 2).

\section{Typical Case Analysis}

1) Target A in Dong Fang zone of Ying Ge Hai basin 


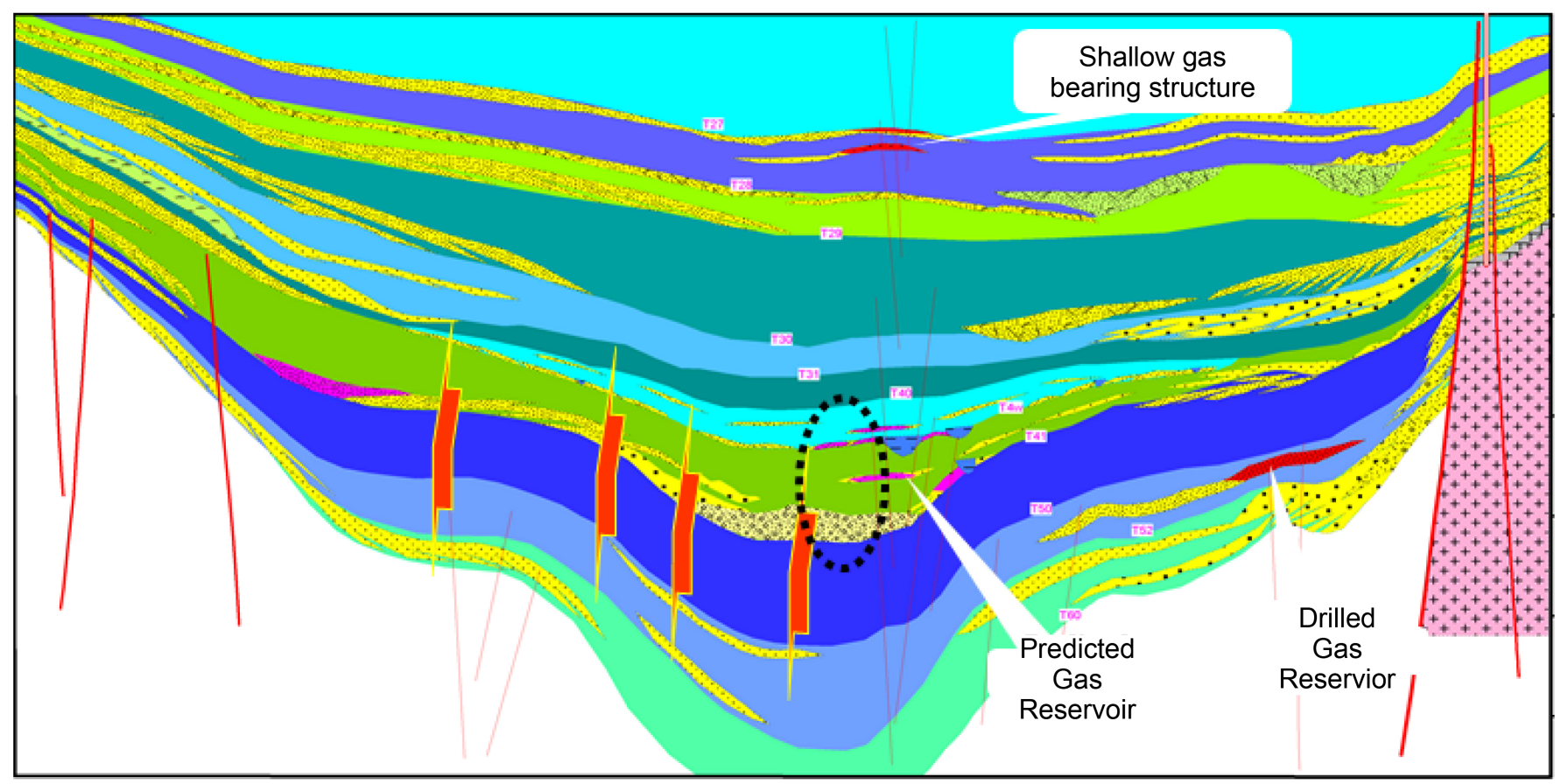

Figure 2. Regional reservoir forming pattern map of target A.

Target A which is located in the central part of Ying Ge Hai basin and near Dong Fang 1-1 gas field belongs to medium deep favorable targets. The main development risk is high pressure. Before pressure prediction, we usually first carefully interpret the velocity spectrum on the design well site or nearby and then make pressure prediction combining with the typical pressure prediction model [13]. In this case, as is shown in the left part of Figure 3, there is an obvious influence of multiple waves and energy clusters on target layers are not concentrated. It leads to more uncertainties of velocity interpretation and cannot meet the requirements of target fine pressure prediction.

In order to solve this problem, the data is reprocessed (Figure 3 right). With more concentrated energy clusters on target layers and less multiple waves, uniqueness of velocity interpretation is better [14] [15] [16]. However, there is a certain velocity error before and after reprocessing. And comparing the two pressure in problem that how to do velocity reprocessing and determine its accuracy.

2) Target B in Bao Dao zone of Qiong Dong Nan basin

Target B is in the Bao Dao zone northeastern of Qiong Dong Nan basin, and located between Bao Dao sag and Song Tao bulge. Before drilling the target, we concern on time-depth conversion problem. Take a drilled well as an example: there is obvious phenomenon of scissors difference between the average velocity calculated by VSP and that obtained from velocity spectrum interpretation. For the tectonic location of target $B$ is similar with this drilled well, the average velocity of Target B obtained from velocity spectrum interpretation is adjusted according to the correction of this well.

After target B is drilled, VSP logging velocity is projected onto the velocity spectrum (Figure 4 left), and it is found that phenomenon of scissors difference between the average velocity calculated by VSP and that obtained from velocity spectrum interpretation also appears (Figure 4 left). The unadjusted velocity has large error compared to 


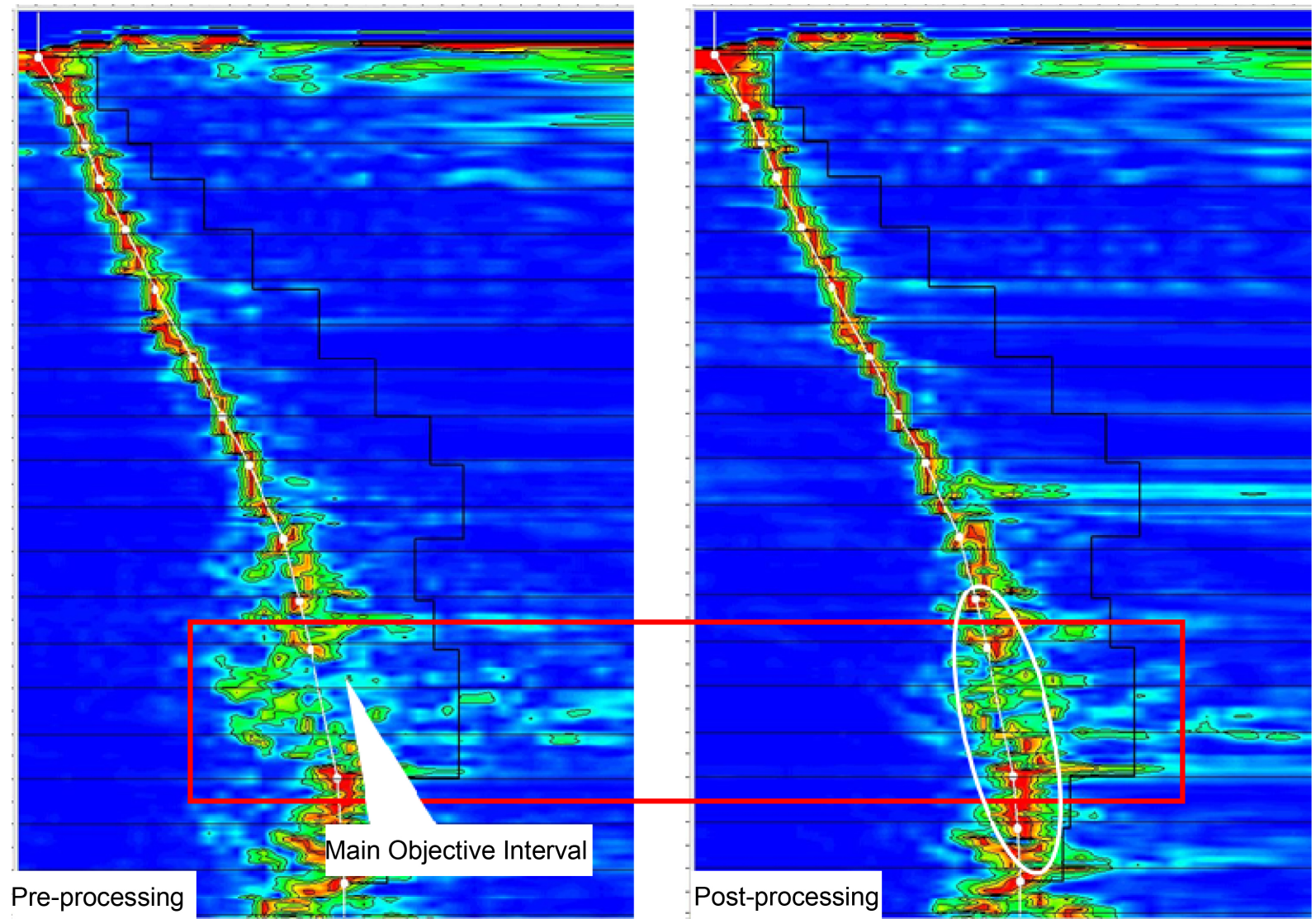

Figure 3. Velocity spectrum comparison before and after reprocessing.
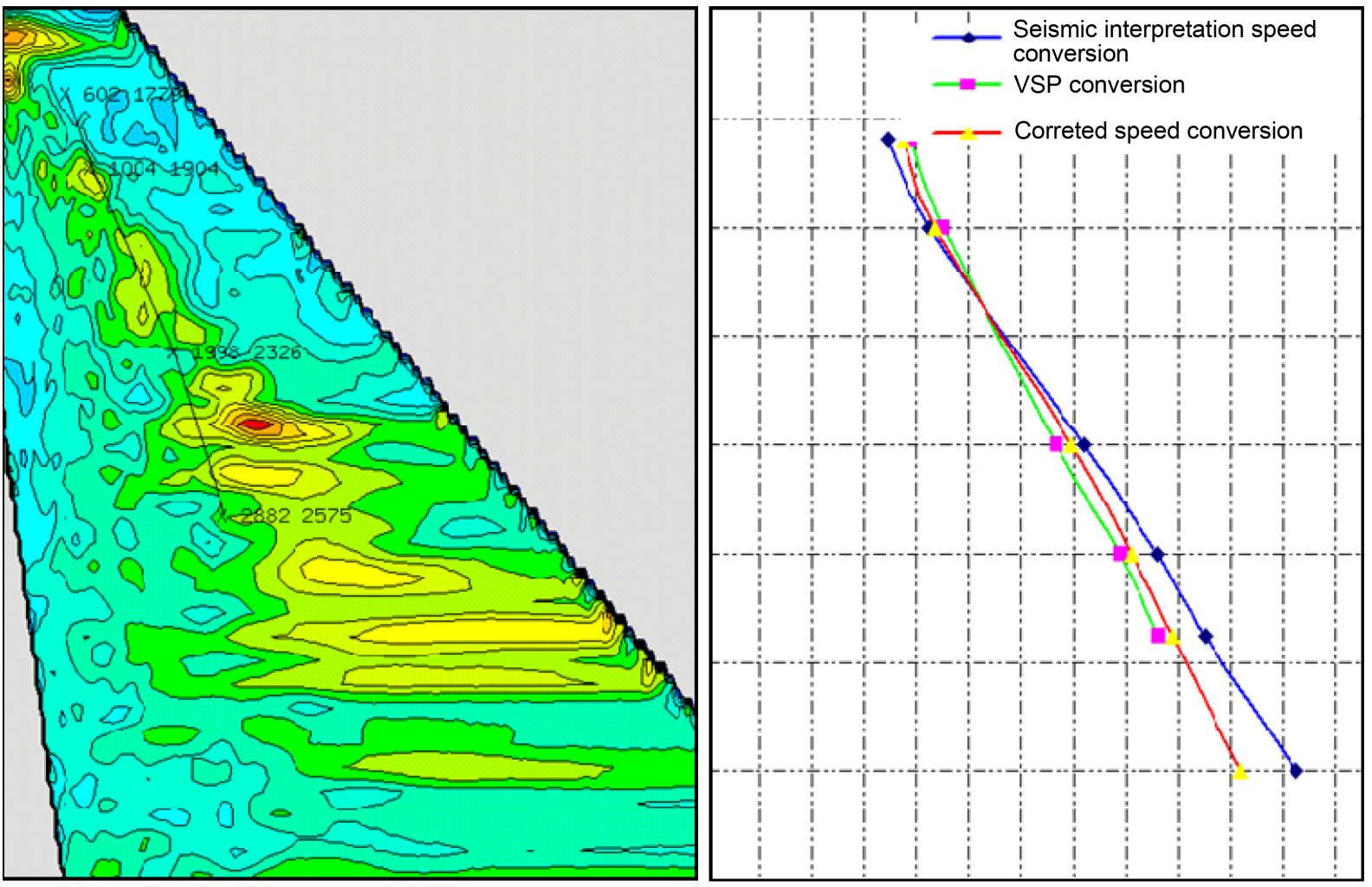

Figure 4. Velocity comparison of target B before and after drilling. 
measured velocity, and though the adjusted velocity is closer, there is still error. Hereby, this example raises the second main problem that the system error exists between logging VSP velocity and velocity spectrum interpretation.

3) Larger error occur when predicting depth of new drilling well with the time-distance relation of drilled wells nearby

Recently HK29B well is drilled in Haikou zone of Ying Ge Hai basin, and BD19C well is drilled in Bao Dao zone of Qiong Dong Nan basin. Both are on high positions of structures. HK29A well and BD19B well are already drilled. Conventional the calibrated time-depth relations of HK29A well and BD19B well are respectively used to predict depths of HK29B well and BD19C well. Meanwhile, fitting velocity formula is used to complete regional time-depth conversion and target layer time-depth conversion, whose trend and amplitude of depth structure are basically the same with that of time domain.

BD19C well and HK29B well were drilled in 2012. By contrast of while-drilling velocity and post-drilling velocity, the same problem of actual depth and predicted depth is observed that large errors (HK29B 10\% and BD19C 12\%) between predicted timedepth relation and actual time-depth relation and the predicted construction highness shifts. Hereby, this example raises the third main problem, namely why there exists large errors when predicting depth of new drilling well with the time-distance relationship of drilled wells nearby and how to deal with similar situations like this afterward (Figure 5).
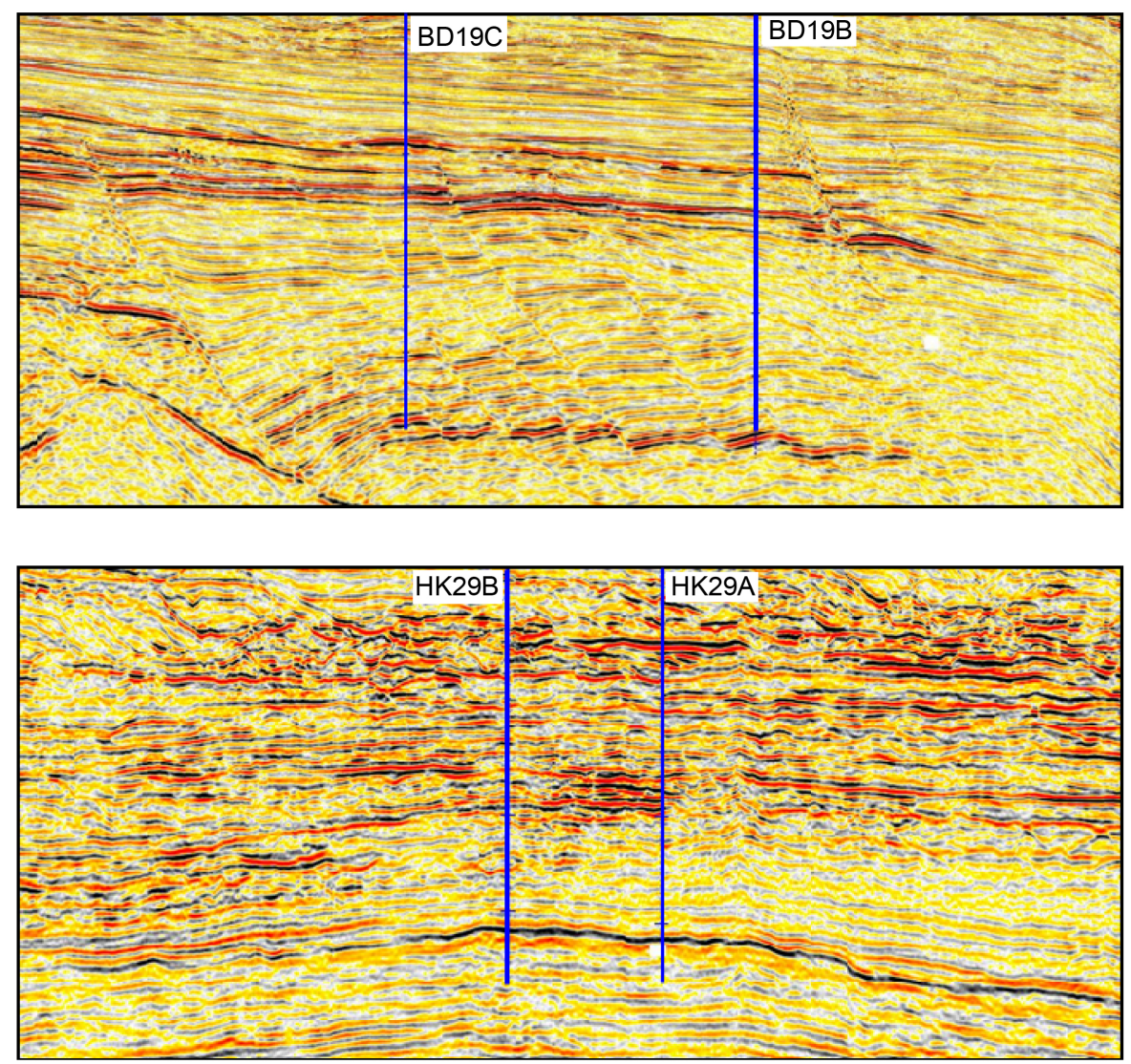

Figure 5. Typical seismic profiles. 


\section{Solution and Method Discussion}

1) Processing velocity with geological model for guidance

In order to answer the first main question, the method that velocity should be processed with geological model for guidance is proposed and a typical example in YGH basin is given to illustrate the idea (Figure 6). There is an obvious influence of multiple waves for medium-deep formation and effective energy clusters are difficult to concentrate. So the velocity spectrum should be reprocessed. As is shown in the upper picture of Figure 6 , the processing outcome is alright when just using radon filtering without considering geological model. However, big difference happens when compared with regional velocity data. The drilled well reveals that a velocity inflection point appears around 1200 $\mathrm{ms}$, but it doesn't appear in this situation. As is shown in the lower picture of Figure 6, when geological model is taken for guidance, the inflection point appears around 1200 $\mathrm{ms}$ and the velocity spectrum changing trends are basically identical with that of logging velocity.
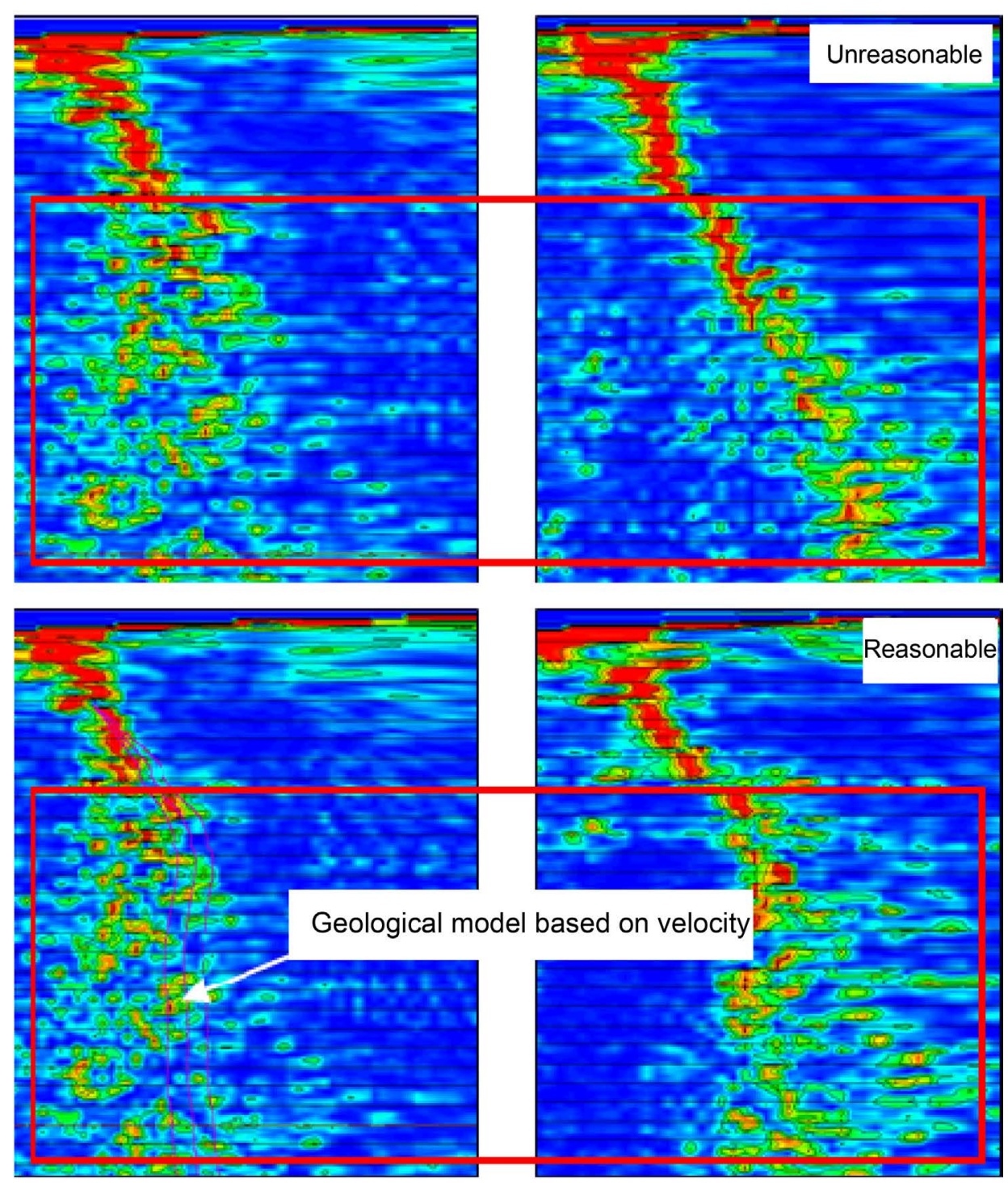

Figure 6. Effect diagram of velocity processed with geological model for guidance. 
2) Establishing regional stratigraphic statics velocity correction templates

As for the second problem, we can see from the second case above that there is phenomenon of scissors difference between the average velocity calculated by VSP and that obtained from velocity spectrum interpretation in different structures of QDN basin. And the differences vary from one block to another. Take QDN basin as an example. To solve this kind of problem, regional stratigraphic statics velocity correction templates of every block in this area are established according to VSP velocity of drilled wells and velocity spectrum crossing the well points. Therefore, the velocity corrected by these templates will be closer to the actual velocity when making new velocity spectrum interpretation in this area in the future.

3) Using along-horizon velocity interpretation and establishing forward modeling in assistance

The velocity spectrum is restricted by its own accuracy, especially when the target layer velocity is abnormally low or high pressure exists. To deal with the third difficulty, two solutions are given. First, project the seismic interpretation horizon in the form of normal velocity into velocity spectrum interpretation work area, and pick up velocity along the horizon (Figure 7). In this way, resulting interval velocity has a certain geological meaning and vertical resolution of the velocity can be increased by interpreting horizon more intensive. Second, build a two-dimensional forward model to assist velocity interpretation. The forward model is based on typical seismic profiles which integrate seismic interpretation horizon with results of along-horizon velocity interpretation. It analyses the tectonic form and amplitude of the target layers in time domain, depth domain and velocity domain to guide the velocity interpretation and abnormal velocity judgment.
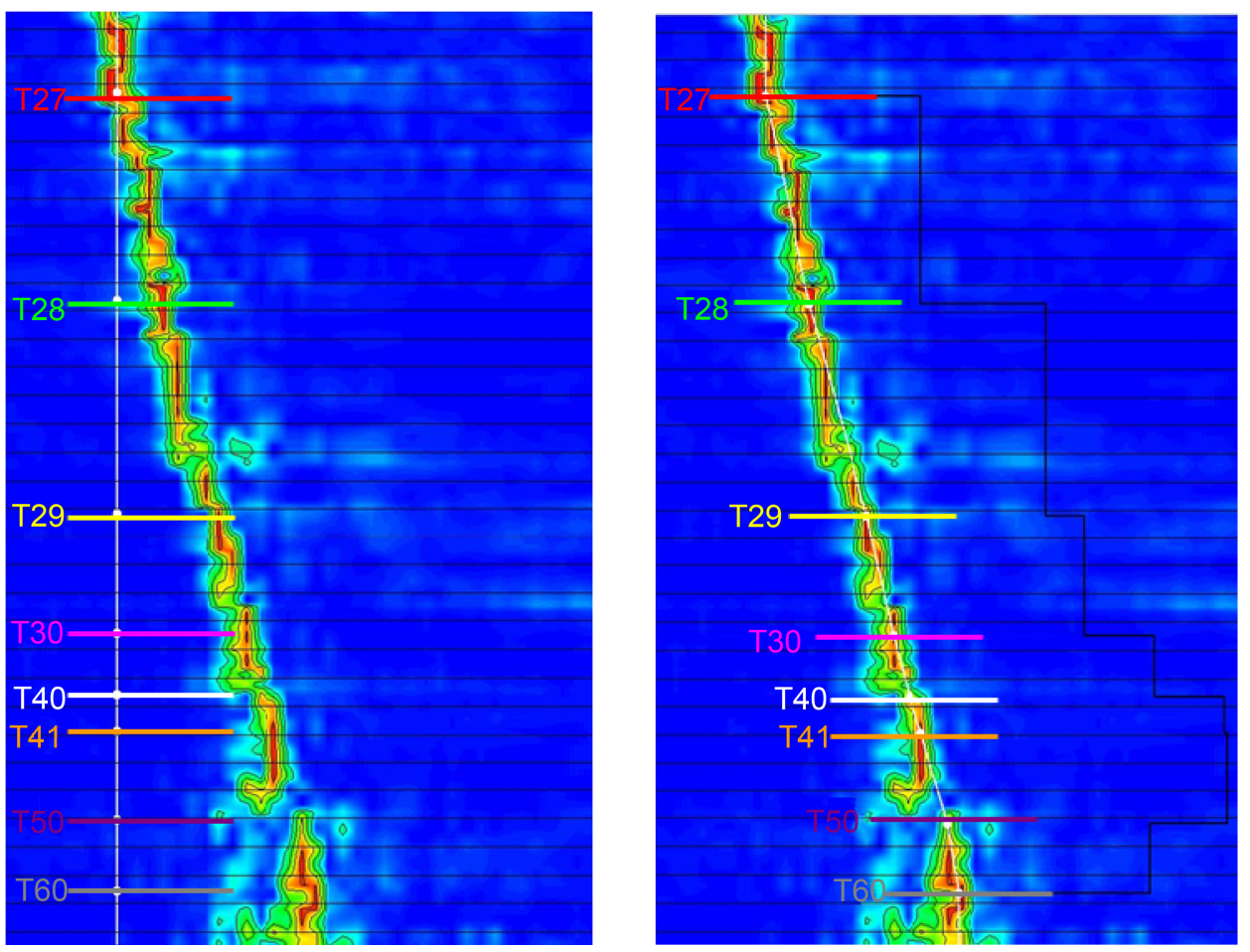

Figure 7. Velocity pick-up along the horizon. 
4) Building the high precision velocity volume with velocity model corrected by information of drilled wells, horizons and geologic zonation

To solve the problem that regional velocity errors exist between new wells and drilled wells at the same structure around the diapir zone, the thinking of building velocity volume and correcting the velocity model is proposed in two steps (Figure 8). Firstly, seismic velocity model is corrected by logging VSP or acoustic velocity curve model and difference of interval velocity is used. Secondly, velocity model obtained in the first step is matched by geological zonation and time zonation, and ratio of interval velocity is adopted. During the process, three quality control points should be focused on. First, make sure the single well VSP or acoustic velocity reasonable, eliminate abnormal data and keep a vertical change tendency as smooth as possible. Second, pay attention to matching of geological layers and time horizons and their closed relationship. Third, compare velocity model error before and after correction, and find out whether the error is smaller and basically in a normal distribution.

\section{Application Analysis}

Velocity model of Dong Fang diapir area in Ying Ge Hai basin is established based on the method above. First, the lateral and vertical variation characteristics of the interval velocity are obvious. The velocity is relatively low at the center of diapir area as for the high pressure, which is consistent with geological knowledge. Then, along-horizon extracted interval velocity is similar to the average velocity lateral variation characteristics and time structure feature. Velocity is significantly less than that at the diapir peripheral, and the interval velocity at the location of DF1A well is significantly higher than that of other surrounding has drilled wells. All these results are consistent with the actual drilling results. Finally, we compare the error before and after correction. Before correcting, the errors of geological zonation vary from $-30 \mathrm{~m}$ to $10 \mathrm{~m}$ and are not in a normal distribution. After correcting, the error margin is from $-3 \mathrm{~m}$ to $3 \mathrm{~m}$, and also meets the normal distribution (Figure 9). The error characteristics prove the velocity correction mode is reasonable.

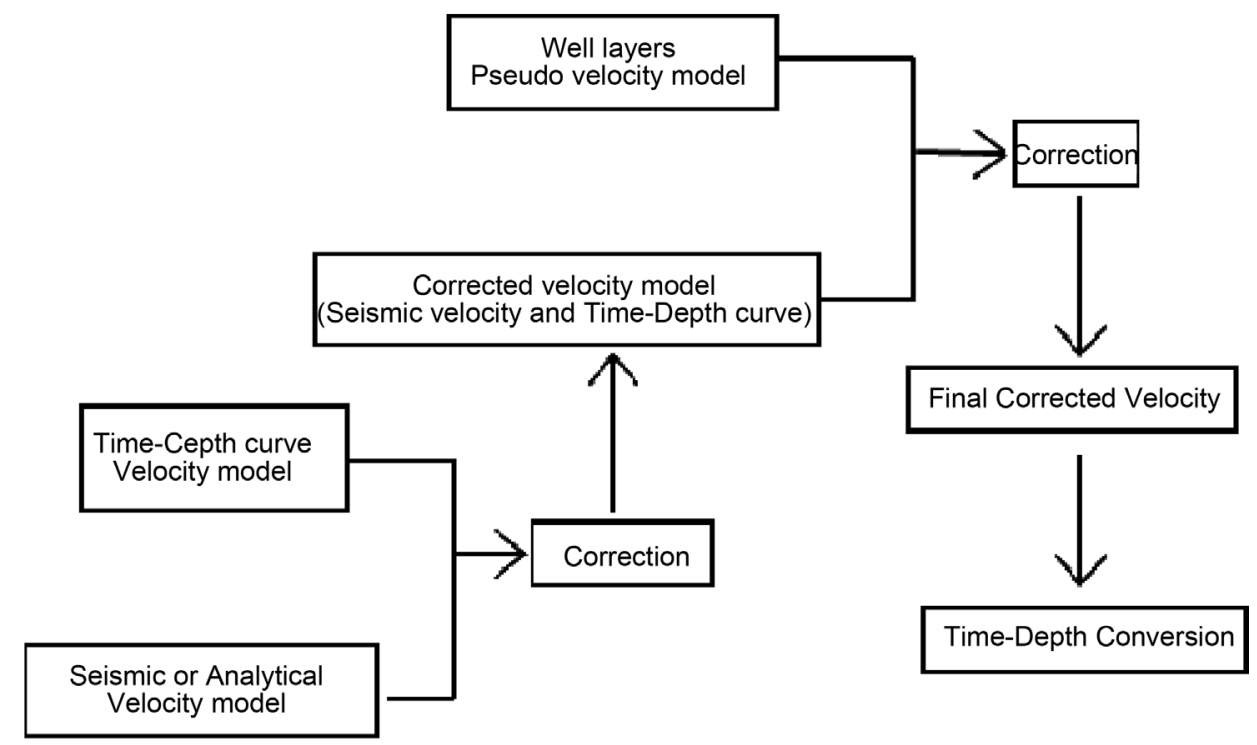

Figure 8. Flow chart of velocity model correction. 

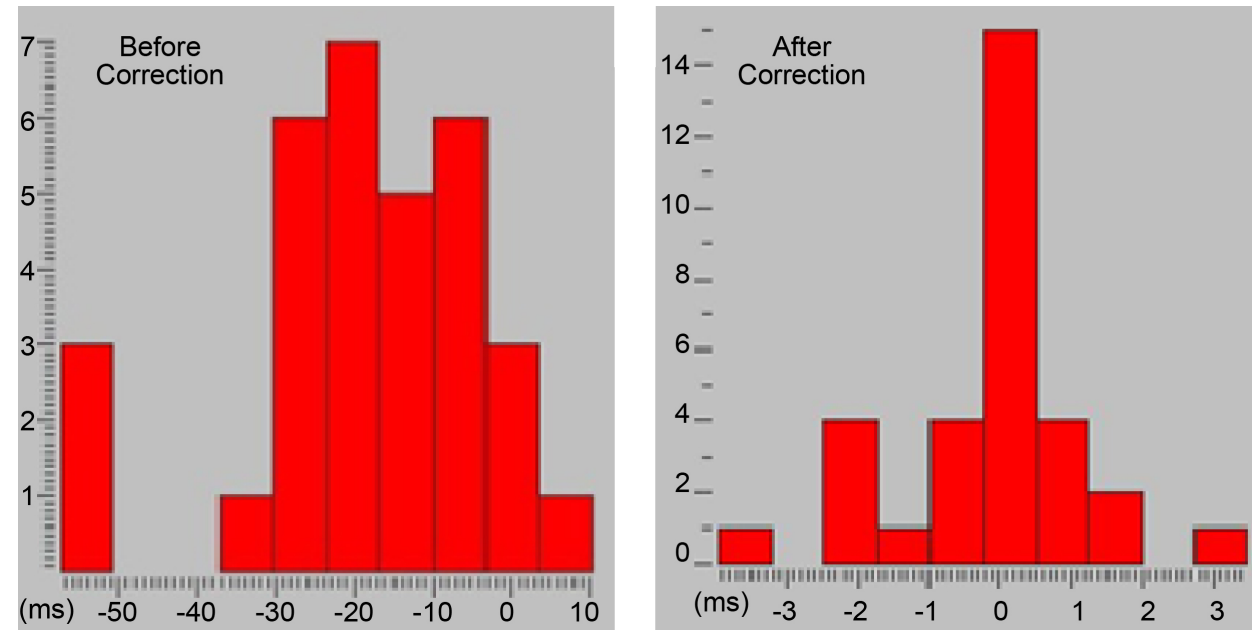

Figure 9. Error comparison histogram before and after velocity correction.

\section{Conclusion and Understanding}

It's very difficult to predict the velocity of favorable targets in a basin with low overall prospecting degree like Ying-Qiong basin. Combined with problems and the misunderstanding, in this paper method research is carried out and corresponding solution is put forward for every problem, which lays a solid foundation for accurate velocity prediction before drilling. First, processing velocity with geological model for guidance solves the problem how to do velocity reprocessing and determine its accuracy. Then, establishing regional stratigraphic statics velocity correction templates diminish the system error existing between logging VSP velocity and velocity spectrum interpretation. Next, using along-horizon velocity interpretation and establishing forward modeling in assistance overcome the difficulty that velocity spectrum is restricted by its own accuracy. Finally, high precision velocity volume is established with velocity model corrected by information of drilled wells, horizons and geologic zonation.

In short, as long as we know more about misunderstandings, overcome the difficulties of velocity analysis and solve the important problems in the process of gas velocity prediction, ideal velocity prediction results can be obtained.

\section{Fund Project}

National Science and technology major special projects supported by 2016ZX05024005.

\section{References}

[1] Chen, X.-R. (1991) An Analysis of Exploration Prospects of YingDong Structure Belt in Yinggehai Basin. Natural Gas Industry, 19, 31-34.

[2] He, J.-X., Xian, Z.-Y., Yang, X.-B. and Mao, Y.-X. (2001) Petroleum Geology and Current Exploration Trend in YingDong Slope Area, Yinggehai Basin, South China Sea. China Offshore Oil and Gas (Geology), 15, 242-248.

[3] He, J.-X., Xia, B., Zhang, S.-L. and Sun, D.-S. (2006) Migration-Accumulation of Gas under the Abnaormal High Geotemperature and Superpressure Circumstance in Yinggehai Basin. Marine Geology \& Quaternary Geology, 26, 81-89.

[4] Li, L., Song, H.-B. and Yang, J.-H. (2006) A Preliminary Study of Seafloor Gas Seepage in 
Central Sag Zone of Yinggehai Basin. Progress in Geophysics, 21, 1244-1247.

[5] Chen, Y., Wei, Y. and Ge, Y. (2004) Multiple Reflection Analysis and Rejection in L Depression of East China Sea. China Offshore Oil and Gas (Geology), 16, 373-376.

[6] Hao, F., Dong, W.-H., Zou, H.-Y. and Yang, X.-S. (2003) Overpressure Fluid Flow and Rapid Accumulation of Natural Gas in Yinggehai Basin. Acta Petrolei Sinica, 24, 7-12.

[7] Cai, G. and Qu, Z.-Y. (2005) Study of Seismic Data Velocity and Mapping Method in Complicated Structure Area and Its Application. Natural Gas Geoscience, 16, 246-249.

[8] Liu, A.-Q., Tong, C.-X. and Li, L. (2008) Research of Velocity Analysis Method and Analysis of Velocity Influence Factors in the East Slope of Yinggehai Basin. Progress in Geophysics, 23, 1909-1917.

[9] Liu, J.-P., Yang, Y.-Q. and Li, X.-Z. (1999) The Method for Extracting Velocity Under the Complex Topographic Condition and its Analysis. Geophysical and Geochemical Exploration, 23, 259-264.

[10] Liu, Q.-F. (2003) Analysis and Application of the Velocity in Imaging of Seismic Data and Mapping of Structure. Journal of Jianghan Petroleum University of Staff and Workers, 17, 52-54.

[11] Liu, T.-Y., Li, Y.-S. and Shi, X.-M. (1995) Geopressure Prediction and Evaluation Methods and its Application in Yinggehai Basin. China Offshore Oil and Gas (Geology), 9, 333-339.

[12] He, J.-X., Li, M.-X. and Huang, B.-J. (2000) The Analysis of the Oil and Gas Exploration Prospect and the Distribution of Outflow of Oil and Gas in the Northern Slope of the Yinggehai Basin. Natural Gas Geoscience, 11, 1-9.

[13] Meng, Q.-S., Chu, X.-F., Guo, X.-J., Fan, Y.-Q. and Jia, Y.-G. (2007) The Application of High Resolution Seismic Data Processing Technique in Multi-Channel Shallow Offshore Engineering Seismic Surveys. Progress in Geophysics, No. 3, 1006-1010.

[14] Tian, S.-C., Chen, Y.-J. and Shi, F.-C. (2004) The Role of Abnormal Pressure Compartment in Oil and Gas Accumulation. Earth Science Frontiers, 11, 283-284.

[15] Xie, R.-J., Zhu, G.-S. and Qi, J.-F. (2003) Application of Sonic Logging in Average Velocity Field. Journal of Southwest Petroleum Institute, 25, 9-12.

[16] Wang, L.-M. and Li, Q.-C. (2006) Improving the Accuracy of Converted-Wave Velocity Analysis with Selected-Correlation Method. Progress in Geophysics, 21, 1213-1220.

\section{Submit or recommend next manuscript to SCIRP and we will provide best service for you:}

Accepting pre-submission inquiries through Email, Facebook, LinkedIn, Twitter, etc.

A wide selection of journals (inclusive of 9 subjects, more than 200 journals)

Providing 24-hour high-quality service

User-friendly online submission system

Fair and swift peer-review system

Efficient typesetting and proofreading procedure

Display of the result of downloads and visits, as well as the number of cited articles

Maximum dissemination of your research work

Submit your manuscript at: http://papersubmission.scirp.org/

Or contact ojms@scirp.org 